\title{
Sputum Quality Challenges the Point of Care Diagnostic System of Pulmonary Tuberculosis in the Republic of Congo
}

\author{
Laure Stella Ghoma Linguissi ${ }^{1 *}$, Genevieve Andoseh ${ }^{1}$, Brigitte \\ Tumamo Fotso ${ }^{1}$, Janine Merveille Mboko Ndangui ${ }^{1}$, Narcisse Arsène \\ Balou ${ }^{1}$, Anne Magalie Murielle Mboungou Nzoussi ${ }^{1}$ and Albert \\ Mabiala $^{2}$ \\ ${ }^{1}$ Institut National de Recherche en Sciences de la Santé (IRSSA), Brazzaville, \\ Republic of Congo \\ ${ }^{2}$ Service des Maladies Infectieuses, Hopital de Référence de Makélékélé, \\ Brazzaville, Republic of Congo \\ *Corresponding Author: Laure Stella Ghoma Linguissi, Institut National de \\ Recherche en Sciences de la Santé (IRSSA), Brazzaville, Republic of Congo.
}

Received: May 03, 2021

Published: July 09, 2021

(C) All rights are reserved by Laure Stella

Ghoma Linguissi., et al.

\begin{abstract}
The quality of sputum specimen remains crucial in the accurate diagnosis of TB. Good quality sputum makes for a high diagnostic yield while poor quality sputum yields false negative results and delays diagnosis. We aimed to determine the effect of sputum quality on smear microscopy results in a referral hospital in the Republic of Congo. The Sputum specimens were collected from suspected pulmonary TB patients and macroscopically observed using set quality parameters and then processed using standard Ziehl-Neelsen (ZN) staining for Acid fast bacilli (AFB). A total of 117 TB suspected patients submitted 229 sputum samples that was processed. Out of the 229 samples, $24.02 \%$ were of good quality (mucopurulent), 1.31\% of average quality (blood tinged) and $72.49 \%$ of poor quality (saliva). There were 61 samples (26.64\%) that tested positive for AFB while 168 (73.36\%) were negative. The positive samples were mainly mucopurulent $88.52 \%$ (54/61), while the negative samples were mainly salivary 97,62\% (164/168). Thus, 37 (31,62\%) patients were diagnosed AFB smear positive (AFB+) and 80 (68.38\%) AFB smear negative (AFB-). Smear negative TB was associated with poor quality sputum (saliva). The results of this study show that the quality of the sputum influences significantly the performance of microscopy for the diagnosis of pulmonary tuberculosis and reveals the fact that inadequate sputum quality compromises the national efforts to control TB. In our study we had a good performance of the macroscopic assessment that identified smear-positive respiratory specimens associated with mucopurulent sputum and smear-negative respiratory specimens associated with saliva. It will be of major importance for health authorities to develop measures that encourage proper and good quality sputum collection for early and accurate pulmonary tuberculosis diagnosis thereby reducing disease transmission within the population.
\end{abstract}

Keywords: Sputum Quality; Acid-Fast Bacilli (AFB); Smear Microscopy; Republic of Congo

\section{Introduction}

Despite tremendous efforts made in the control of Tuberculosis, the disease still holds many victims captive worldwide especially in developing countries. According to the WHO, 10 million active cases of TB were diagnosed in 2018 and a total of 1.2 million died 
from the disease [1]. An efficient TB diagnosis is made by demonstrating the presence of tubercle bacilli in the specimen collected by means of microscopy and/or culture in the laboratory. Culture is the gold standard for diagnosing pulmonary tuberculosis of a sputum specimen. However, due to lack of access to culture facilities and the long turn-around times involved with sputum culture, sputum microscopy remains the mainstay of diagnosis for acid-fast bacilli (AFB) and treatment follow up because of its availability, operational feasibility and ability to identify the highly infectious forms of TB in many developing countries [2,3] including Congo Brazzaville.

Sputum smear microscopy, although quick and easy to perform, suffers from low specificity and sensitivity and the quality of sputum plays a major role in the quality of the microscopy result $[2,4-$ 7]. Examining the specimen quality before diagnosis by a skilled laboratory staff is essential to diagnose TB accurately and avoiding missed cases. Generally, sputum provided for AFB examination is first assessed macroscopically by laboratory staff in many TB diagnostic centers.

A visual acceptance of a good quality sputum consists of recently-expectorated biological material from the bronchial tree, with minimum amounts of oral or nasal material but a greater presence of mucoid or mucopurulent material. Poor quality specimens are thin and watery or composed largely of bubbles and they are grouped under the name saliva [3]. If the quality of the sputum is deemed inadequate, the specimen is rejected and the TB suspect asked to provide a new specimen. Rejecting a poor-quality specimen and asking a patient to provide another new sample is considered difficult by many TB suspects. The risk exists that the TB suspect will not return at a later stage to provide a sputum specimen leading to a missed opportunity for TB case finding [3-8].

Patients frequently provide pure saliva or very small amounts of sputum in saliva instead of an appropriate, purulent, sputum [3] specimen. Saliva smears mostly generate negative results which could be misleading for different specimens, given that a correct sputum specimen has not been examined. On the other hand, saliva-containing sputum specimens could also contribute to the diagnosis of TB [4]. However, there is limited information about the contribution of sputum quality in microscopy diagnosis in Congo. Hence our study aimed to compare the quality of sputum speci- mens collected from different TB suspect patients and the microscopy result obtained in a hospital setting.

\section{Materials and Methods}

Study design

It concerns a cross-sectional study carried out throughout 2019 in the infectious diseases department of the Makelekele referral hospital, in Brazzaville, Republic of the Congo. Participants with suspected pulmonary tuberculosis were enrolled consecutively to assess the association of sputum samples produced, sputum quality, and smear microscopic examination issuance.

\section{Sputum collection}

Sputum specimens were collected from 117 suspected pulmonary TB patients (never been treated for TB or have received anti tubercular treatment for $<1$ month). Two consecutive sputum samples from each patient (home: early morning sputum sample collected at home is called as home sample) were collected over two days. Currently, the guidelines of the National Tuberculosis Control Program recommend the use of a single jar of sputum for the tuberculosis screening center which has been provided with GeneXpert, this is the case of the Anti-tuberculous Center of Brazzaville $[9,10]$. The specimens were then characterized macroscopically by skilled laboratory staff.

Macroscopic characterization of sputum and Ziehl Neelsen Microscopy

This was done as previously described by Yoon and colleague with some adaptation [11]. Sputum that had mucoid and purulent material was categorized as muco-purulent and graded good quality. The sputum specimens having reddish/rusty color was labeled as blood-tinged sputum and graded average quality. Sputum that had clear and watery appearance without any viscosity was categorized as saliva and graded poor quality. Whenever the sputum specimens were heterogeneous, the predominant portion was considered to be the quality of sputum specimen.

All the samples were subjected to routine Ziehl Neelsen (ZN) staining. AFB strains were classified according to the WHO/International Union against Tuberculosis and Lung Disease scale: $1+(10$ - 99 AFBs per 100 fields), 2+ (1 - 10 AFBs per individual field), 3+ (10 - 100 AFBs per individual field), $4+(>100$ AFBs per individual field) and Negative [12]. 


\section{Statistical methods}

Frequencies and proportions were used to analyze categorical variables. Pearson's Chi-square was used at bivariate level to test for the association between smear result (positive and negative) and sputum quality. Odds ratio (OR) with 95\% CI was used to determine the magnitude/strength of the association.

Ethics

Human subjects' approval was granted by the administrative hospital committee. Written informed consent was obtained from all eligible study participants and witnessed by the study clinician.

\section{Results}

A total of 117 suspected TB patients were enrolled and 229 home sputum specimens were collected. Among them, 112 patients provided two sputum's samples and 5 patients provided one sample.

Out of the 117 patients, 37 (31.62\%) patients were diagnosed AFB smear positive (AFB+) and 80 (68.38\%) AFB smear negative (AFB-) (Table 1). The group of patients diagnosed with AFB+ was on the basis of the fact that at least 1 of the 2 sputum samples sub- mitted was positive. The group of patients diagnosed with AFBwas on the basis of the fact that both sputum samples submitted were negative.

The 229 sputum samples submitted had the following characteristics: $24.02 \%$ (55/229) mucopurulent, 1.31\% (3/229) blood tinged, $72.49 \%$ (166/229) saliva, and 2.18 (5/229) missing.

In the group of $\mathrm{AFB}+$ diagnosed patients, 36 submitted 2 samples, 1 patient submitted 1 sample making a total of 73 samples analyzed. Of these 73 samples, 62 total sputum samples were positive at microscopy, 11 were negative. Amongst the 62 positive sputum samples, 55 (88.71\%) were mucopurulent; 1(1.61\%) was blood, 2 $(3.23 \%)$ were saliva, and $4(6.45 \%)$ did not have the sputum quality specific. Amongst the 11 negative sputum samples, all (100\%) were saliva.

In the group of AFB- diagnosed patients, 76 of these patients submitted 2 samples and 4 patients submitted 1 sample making a total of 156 samples analyzed. Of these 156 samples, all were negative at microscopy. Amongst these 156 negative sputum samples, $153(98.08 \%)$ were saliva and $2(1.28 \%)$ were blood and $1(0.64 \%)$ did not have the sputum quality specific.

\begin{tabular}{|l|c|c|c|c|c|}
\hline \multirow{2}{*}{$\begin{array}{c}\text { Quality of sputum } \\
\text { submitted }\end{array}$} & \multicolumn{2}{|c|}{$\begin{array}{c}\text { AFB+ } \\
\text { (N= 37, n = 73) }\end{array}$} & $\begin{array}{c}\text { AFB- } \\
\text { (N = 80, } \mathbf{n}=156)\end{array}$ & \multicolumn{2}{c|}{$\begin{array}{c}\text { All Sputum samples } \\
\text { (n= 229) }\end{array}$} \\
\cline { 2 - 6 } & Positive result & Negative result & Negative Result & Positive result & Negative result \\
\hline Mucopurulent & 55 & 0 & 0 & 55 & 0 \\
\hline Blood tinged & 1 & 0 & 2 & 1 & 2 \\
\hline Saliva & 2 & 11 & 153 & 2 & 164 \\
\hline Missing & 4 & 0 & 1 & 62 & 1 \\
\hline Total & 62 & 11 & 156 & & 167 \\
\hline
\end{tabular}

Table 1: Microscopy results of sputum samples submitted by patients and graded with different quality characteristics categorized under AFB+ and AFB-.

$\mathrm{N}$ : it represents the number of patients diagnosed within each category (AFB+ and AFB-); n: it represents the number of sputum samples submitted and analyzed.

Out of the 229 sputum samples submitted, 62 (27.07\%) were positive for AFB while 167 (72.93\%) were negative (Table 1). The positive samples were characterized by mucopurulent $88.71 \%$ $(55 / 62)$, blood tinged $1.61 \%(1 / 61)$ and saliva $3.23 \%(2 / 62)$.
The negative samples were characterized by blood tinged $1.20 \%$ (2/167) and saliva 98.20\% (164/167).

Amongst the 62 positive sputum samples, 55 (88.71\%) were mucopurulent; $1(1.61 \%)$ was blood tinged, 2 (3.23\%) were saliva, 
and $4(6.45 \%)$ did not have the sputum quality specific. Amongst the 11 negative sputum samples, all (100\%) were saliva.

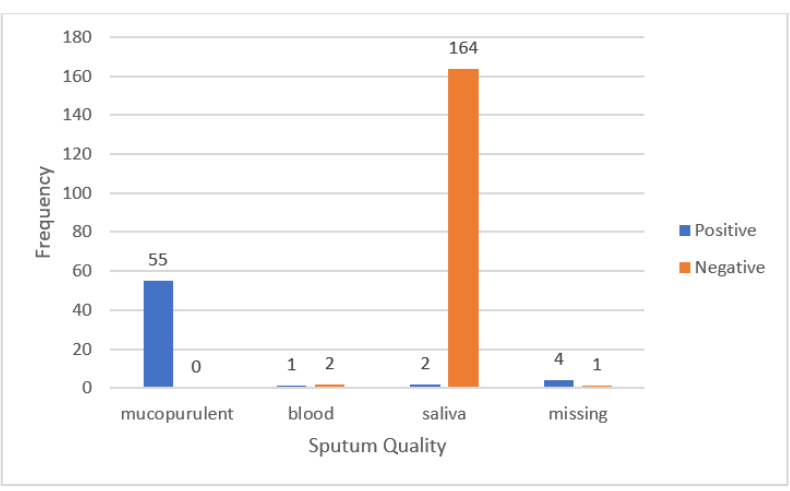

Figure 1: Association of sputum characteristics with positive and negative microscopy results.
In the group of patients diagnosed with $\mathrm{AFB}+(37 / 117), 5.41 \%$ (2/37) were HIV + and 94.59\% (35/37) were HIV negative. Of the 2 HIV positive patients that submitted 2 sputa each, 1 patient had the quality missing for the sputa. Concerning the other patient who submitted 2 sputa, 1 sputum was mucopurulent and the smear result was positive while the other was saliva and the smear result was negative. Amongst the AFB+ and HIV negative group, positive samples were characterized by mucopurulent $71.41 \%$ (54/68), blood tinged $1.47 \%$ (1/68) and saliva 2.94\% (2/68) samples that were positive. There were 10 salivary samples that were all negative.

In the group of patients diagnosed with AFB- (80/117), 5.41\% (22/80) were HIV positive and 94.59\% (58/80) were HIV negative. Amongst the HIV positive patients, there was a total of 42 samples submitted. All 42 samples were salivary in nature and the smear result was negative. Amongst the HIV negative patients, 1 patient submitted 2 blood-tinged samples and they were negative. There

\begin{tabular}{|c|c|c|c|c|c|c|}
\hline \multirow{2}{*}{$\begin{array}{l}\text { Quality of } \\
\text { sputum } \\
\text { submitted }\end{array}$} & \multicolumn{2}{|c|}{$\begin{array}{l}\text { AFB+/HIV+ } \\
(N=2, n=4)\end{array}$} & \multicolumn{2}{|c|}{$\begin{array}{c}\text { AFB }+/ \text { HIV- } \\
(N=35, n=68)\end{array}$} & \multirow{2}{*}{$\begin{array}{c}\text { AFB-/HIV+ } \\
(\mathrm{N}=22, \mathrm{n}=42) \\
\text { Negative result }\end{array}$} & \multirow{2}{*}{$\begin{array}{c}\text { AFB-/HIV- } \\
(\mathrm{N}=58, n=115) \\
\text { Negative result }\end{array}$} \\
\hline & $\begin{array}{c}\text { Positive } \\
\text { result }\end{array}$ & $\begin{array}{l}\text { Negative } \\
\text { result }\end{array}$ & $\begin{array}{c}\text { Positive } \\
\text { result }\end{array}$ & $\begin{array}{l}\text { Negative } \\
\text { result }\end{array}$ & & \\
\hline Mucopurulent & 1 & 0 & 54 & 0 & 0 & 0 \\
\hline Blood tinged & 0 & 0 & 1 & 0 & 0 & 2 \\
\hline Saliva & 0 & 1 & 2 & 10 & 42 & 112 \\
\hline Missing & 2 & 0 & 1 & 0 & 0 & 1 \\
\hline Total & 3 & 1 & 58 & 10 & 42 & 115 \\
\hline
\end{tabular}

Table 2: Association of HIV status amongst AFB+ and AFB- to the quality of sputum submitted and resultant microscopy results. $\mathrm{N}$ : It represents the number of patients diagnosed within each category; $\mathrm{n}$ : it represents the number of sputum samples submitted and analyzed.

were more salivary samples in this group making up to $97.39 \%$ $(112 / 115)$ and all were negative.

\section{Discussion}

Sputum smear microscopy still remains the cornerstone of diagnostic algorithms in national TB control programs in low- and middle-income settings due to its low cost and ubiquitous availability despite recent advances in methods to diagnose TB [13]. The quality of the sputum affects the diagnostic accuracy $[6,8]$. In the present study, we assessed the sputum quality with regard to smear microscopy results. A total of 117 suspected TB patients were enrolled and 229 home sputum specimens were collected. Out of the 229 samples, $24.02 \%$ were of good quality (mucopurulent), $1.31 \%$ of average quality (blood tinged) and $72.49 \%$ of poor quality (saliva). The fact that majority of the samples had the quality mentioned reflects a good observance of the macroscopic identification of the submitted sputum samples. However, this high percentage of poor-quality sputum reveals an absence of salivary specimen rejection in the hospital setting which demonstrates the precaution taken by laboratory technicians not to reject sputum 
that is submitted for AFB smear [8]. According to [5], the prevailing practice of testing all sputum submitted in the laboratory should continue since it takes only one sputum smear to make a diagnosis in countries that have an established external quality assurance system.

One reason for the high proportion of poor-quality samples could be that patients don't really understand how to produce good quality sputum. We did not investigate from patients if they received instructions. In the study carried out by [14], they studied the factors associated with insufficient quality of sputum. Out of 387 TB suspects, they had information from 272 whose sputum quality was available. Of the 272 TB suspects, 168 (61.8\%) mentioned that information on the reason for sputum examination was provided, 66 (24.3\%) remembered that they were informed about how to produce sputum and $40(14.7 \%)$ recalled being informed about the characteristics of good quality sputum. They found that information on how to produce sputum and characteristics of a good sputum sample was less often provided [14]. A research group in Pakistan has developed a sputum submission instructional video for improving the diagnostic quality of sputum samples (available at http://youtu.be/92dT_1kbbek or http://vimeo.com/ irdresearch/good-sputum-english) which was associated with detection of a higher proportion of microscopically confirmed cases [15]. The use of such a culturally adapted instructional video for sputum submission in our setting could be implemented and evaluated for its impact in providing good quality specimens and fostering TB diagnosis. In fact, video instructions was found to be associated with improved odds of positive microscopy compared to verbal instructions giving with diagrams [16].

It could also be that the laboratory technicians do not want to reject the saliva sample for fear of the fact that the patient may not come again. This usually occurs when patient brings in the sample in the absence of the lab technician thus hampering a quick macroscopic assessment of sputum quality. If a macroscopic assessment is done as soon as patient hands over sample, sputum collection could be repeated by patient in order to get a good sputum quality [6]. Another reason for poor quality sputum could be linked to patient difficulty in producing good sputum especially in people living with HIV/AIDS.

Out of the 117 patients, 37 (31.62\%) patients had smear-positive TB and 80 (68.38\%) had smear negative TB. In the group of pa- tients with smear positive TB, the predominant sample quality type was mucopurulent $(88.71 \%)$ which correlated with the positive smear results. Only 2 (3.23\%) salivary samples in this group were positive. The rest were negative. The AFB positivity rate $(3.23 \%)$ of saliva in our study is lower than that of Yoon., et al. (17.6\%, 19 of 108). Despite the low positivity rate, saliva-like sputum specimens should not be discarded because they contribute in the diagnosis of TB $[4,11]$. In the work carried out by [17] they used the criterion of McCarter and Robinson (1996) developed for AFB-screening of specimens and found that out of $13 \%$ of rejected samples, $3 \%$ were AFB-positive. It is important to test all samples received so as not to miss any potential AFB positive specimen.

In the current study, a considerable number of patients were negative to ZN smear microscopy. In the group of patients with smear negative TB, 153 samples (98.08\%) were saliva. There was agreement in the tendency of saliva samples to be AFB-negative. This high proportion of smear negative results is due to the poorquality type (saliva) used in the diagnosis.

It was also observed that 2 samples (1.28\%) of average quality (blood tinged) were negative at microscopy. We did not explore reasons why blood-stained sputum which is not poor quality was smear negative. A potential explanation for lower sensitivity could be that the major visual observation was blood but in terms of fluid content, was not mucus but saliva thus reducing diagnostic sensitivity.

This study has exposed some issues with the microbiological identification of the bacilli using smear microscopy. It is highly likely that poor sputum quality leads to underdiagnosis by smear microscopy in the Republic of Congo which may predispose it to active transmission. Apart from this, culture, the current nucleic acid amplification tests such as Xpert MTB/RIF, Line Probe assay etc. are sputum-based tests used for TB diagnosis and detection of multi drug resistant TB (MDR TB) patients and poor quality sputum will also affect their effectiveness $[6,8]$. However, the work of Meyer and colleagues suggested that salivary sputum although not appropriate for smear microscopy, may provide greater sensitivity than other samples when tested with Xpert but they did not explore the reasons behind it [18].

It has been shown that people living with HIV usually have smear-negative pulmonary and extra-pulmonary tuberculosis and 
that the quality of specimen must also be optimum [8]. There were 2 patients (5.41\%) with HIV who had smear positive TB. Of the 2 HIV positive patients, only 1 had the quality of sputa mentioned. Thus, 1 sputum was mucopurulent and the smear result was positive while the other was saliva and the smear result was negative. Still, positivity was due to the good sputum sample analyzed. In the group of patients with smear negative TB, 5.41\% (22/80) were HIV positive who submitted 42 samples. All 42 samples were salivary in nature and the smear result was negative. Even though it is well established that TB diagnosis in HIV infected people is complicated by their inability to cough deeply from the lung and produce sputum due to impaired immunity but rather produce saliva resulting to higher rates of sputum smear-negative TB [18-20], a greater population of those who had smear negative TB were HIV negative (94.59\%) and they submitted poor quality sputum. Still, we associated the negative results with the quality of sputum submitted irrespective of being infected with HIV or not. Out of the 80 patients with smear negative TB, 37 were put on antituberculosis treatment on the basis of their clinical symptoms and chest $\mathrm{X}$ ray findings. Since they are smear negative symptomatic cases, it is probable that microscopic diagnosis might have missed due to the poor quality of sputum submitted.

There are some limits to the study. In assessing the quality of the sputum samples submitted, it was only evaluated macroscopically. There is a microscopic assessment of Gram stained specimens recording Neutrophils and Squamous Epithelial Cells (SEC) from three consecutive fields for considerations of good and unsatisfactory quality $[4,6,8]$ that was not performed. Other risk factors associated with the quality sputum such as age, gender, educational status was not evaluated in this study.

\section{Conclusion}

A considerable number of TB suspects provided poor quality sputum that affected the detection of smear positive TB. Training of health workers in providing health education to the TB suspect about the reason for sputum examination and how to produce a good quality sputum sample should be a priority in the national TB program. This will limit transmission of the disease with timely diagnosis and proper administration of antimicrobial agents.

\section{Acknowledgements}

We thank the health workers working in the infectious diseases department for their collaboration, the General Management of the Makélékélé Reference Hospital for authorizing this study, and to patients who voluntarily participated in the study for allowing their samples to be used for science.

\section{Bibliography}

1. World Health Organization. "Global tuberculosis report 2019". Geneva: World Health Organization; (2019).

2. Nagabhushan Vidya NV., et al. "Comparative analysis of tuberculosisbacteria in saliva and sputum using Ziehl-Neelsen, Papanicolaou and bleach modified Papanicolaou induced fluorescent microscopy". World Journal of Current Medical and Pharmaceutical Research 8 (2020): 258-266.

3. Banu S. "Comparison of Macroscopic and Microscopic Assessment of Specimens Collected for the Diagnosis of Tuberculosis". Open Infectious Diseases Journal 6.1 (2012): 1-4.

4. Lee YJ., et al. "Acceptability of Sputum Specimens for Diagnosing Pulmonary Tuberculosis". Journal of Korean Medical Science 30.6 (2015): 733.

5. Ho J., et al. "The impact of sputum quality on tuberculosis diagnosis: a systematic review". The International Journal of Tuberculosis and Lung Disease 19.5 (2015): 537-544.

6. Aparna L M., et al. "Assessment of Sputum Quality and Its Importance in the Rapid Diagnosis of Pulmonary Tuberculosis". Archives of Clinical Microbiology 8.4 (2017).

7. Budayanti NS., et al. "The Quality of Sputum Specimens as a Predictor of Isolated Bacteria From Patients With Lower Respiratory Tract Infections at a Tertiary Referral Hospital, Denpasar, Bali-Indonesia”. Frontiers in Medicine 5.6 (2019): 64.

8. Orina F., et al. "Intrinsic and extrinsic factors associated with sputum characteristics of presumed tuberculosis patients". Plos One 14.12 (2019): e0227107.

9. Okemba Okombi FH., et al. "Extensively resistant tuberculosis: Four cases in the Republic of Congo". Medecine et Sante Tropicales 28.3 (2018): 289-291.

10. Okemba-Okombi FH., et al. "Apport du GeneXpert dans le diagnostic de la tuberculose pulmonaire : étude préliminaire et perspectives en République du Congo". Revue des Maladies Respiratoires 32 (2015): A211. 
11. Yoon SH., et al. "Impact of sputum gross appearance and volume on smear positivity of pulmonary tuberculosis: a prospective cohort study". BMC Infectious Disease 12.1 (2012): 172.

12. Linguissi LS., et al. "Prevalence of national treatment algorithm defined smear positive pulmonary tuberculosis in HIV positive patients in Brazzaville, Republic of Congo". BMC Research Notes 7.1 (2014): 578.

13. Dheda K., et al. "Point-of-care diagnosis of tuberculosis: Past, present and future: POC TB diagnostics review". Respirology 18.2 (2013): 217-232.

14. Sakundarno M., et al. "Insufficient quality of sputum submitted for tuberculosis diagnosis and associated factors, in Klaten district, Indonesia”. BMC Pulmonary Medicine 9.1 (2019): 16.

15. Mhalu G., et al. "Do Instructional Videos on Sputum Submission Result in Increased Tuberculosis Case Detection? A Randomized Controlled Trial”. Plos One 10.9 (2015): e0138413.

16. Datta S., et al. "Comparison of sputum collection methods for tuberculosis diagnosis: a systematic review and pairwise and network meta-analysis". Lancet Global Health 5.8 (2017): e760-771.

17. Khan MS., et al. "Judging respiratory specimen acceptability for AFB microscopy: visual vs. microscopic screening". Tropical Medicine and International Health 14.5 (2009): 571-575.

18. Meyer AJ., et al. "Sputum quality and diagnostic performance of GeneXpert MTB/RIF among smear-negative adults with presumed tuberculosis in Uganda". Plos One 12.7 (2017): e0180572.

19. Asemahagn MA., et al. "Determinants of Sputum Smear Positivity among Tuberculosis Suspected Patients in Bahir Dar City, Northwest Ethiopia". Nursing and Health Sciences (2018).

20. Campos LC., et al. "Characteristics of Patients with Smear-Negative Pulmonary Tuberculosis (TB) in a Region with High TB and HIV Prevalence". Plos One 11.1 (2016): e0147933.

Volume 4 Issue 8 August 2021

(c) All rights are reserved by Laure Stella Ghoma Linguissi., et al. 